Meta

Journal des traducteurs

Translators' Journal

\title{
Linguistique différentielle et traduction
}

\section{Jean Darbelnet}

Volume 16, numéro 1-2, mars 1971

Actes du colloque international de linguistique et de traduction.

Montréal, 30 septembre - 3 octobre 1970

URI : https://id.erudit.org/iderudit/002481ar

DOI : https://doi.org/10.7202/002481ar

Aller au sommaire du numéro

Éditeur(s)

Les Presses de l'Université de Montréal

ISSN

0026-0452 (imprimé)

1492-1421 (numérique)

Découvrir la revue

Citer cet article

Darbelnet, J. (1971). Linguistique différentielle et traduction. Meta, 16(1-2),

17-24. https://doi.org/10.7202/002481ar d'utilisation que vous pouvez consulter en ligne.

https://apropos.erudit.org/fr/usagers/politique-dutilisation/ 


\section{Linguistique différentielle et traduction}

Le but de cette communication est de rappeler ce qu'est la linguistique différentielle et de montrer en quoi elle peut aider à la formation du traducteur. Le terme même de linguistique différentielle n'est pas encore très répandu. On parle depuis longtemps de grammaire comparative et depuis quelque temps de contrastive linguistics. La grammaire comparative a surtout comme champ d'étude les langues anciennes; elle est diachronique. Quant au terme contrastive linguistics, il devrait vouloir dire la même chose que linguistique différentielle. Cependant on constate que les linguistes américains qui s'occupent de contrastive linguistics partent des structures des langues étudiées, alors que je conçois la grammaire différentielle comme l'étude des réalisations grammaticales, dans deux langues données, d'un concept qui leur est commun, tel que la caractérisation, la représentation, l'antériorité dans le passé, l'indiscrimination, etc.

Quand on aborde la linguistique différentielle dans les rapports avec la traduction, il n'est que juste de rappeler que l'on n'a pas attendu cette nouvelle discipline pour faire de bonnes traductions. Mais il est évident que l'extension qu'a prise la traduction dans le monde d'aujourd'hui et l'accroissement du nombre des traducteurs, qui en découle, nous obligent à prévoir une formation méthodique préparant à cette profession. Le temps n'est plus où l'on pouvait s'improviser traducteur sous prétexte qu'on savait deux langues. Les traducteurs de génie, qui n'ont pas besoin d'être formés, sont trop peu nombreux pour suffire à nos besoins croissants.

Si l'on admet la nécessité de cette formation, il est facile de voir pourquoi, de par sa nature même, la linguistique différentielle doit y occuper une place importante. Si les langues étaient des codes aux unités interchangeables, cette discipline n'existerait pas. Elle existe parce que les langues ne découpent pas la réalité de la même façon, ne mettent pas les mêmes structures au service des mêmes significations et donnent souvent un sens différent à des structures parallèles ou identiques.

L'une des leçons qu'enseigne presque tout de suite la linguistique différentielle, c'est que la traduction littérale représente un danger pour l'intégrité de la langue d'arrivée et même pour le sens du message. Le littéralisme a sévi au 
Canada pendant de longues années, il y sévit encore et il n'est sans doute pas inconnu ailleurs. Beaucoup de traducteurs, surtout lorsqu'ils débutent, se raccrochent au mot à mot comme à leur planche de salut. Il leur semble qu'on ne peut pas se tromper quand on colle au texte et ils sont même un peu incrédules quand on leur montre que la traduction littérale est parfois un contresens. Il leur faut apprendre que la syntaxe a ses faux amis tout comme le vocabulaire. Peut-être devrait-on commencer par bien faire saisir aux étudiants de traduction cette vérité essentielle que dans le passage d'une langue à l'autre la pensée et la structure ne restent pas forcément liées. C'est ainsi qu'il arrive qu'on change le sens en gardant la structure et qu'il faille changer de structure pour garder le sens. Dans mon cours de «Pratique et théorie de la traduction», j'ai commencé, cette année, par montrer qu'il en est ainsi dans une seule langue. J'ai utilisé à cette fin un exemple que donne R.-L. Wagner dans son petit livre sur les Vocabulaires français (Didier, 1967) : La tempête qui a sévi la semaine dernière a ravagé l'Europe - L'homme qui a dit cela a trompé son public. Ces deux phrases, comme le fait remarquer Wagner, ont la même structure, ou presque. Elles comportent un terme initial déterminé, une relative qui définit, et un prédicat verbal avec complément. De plus, tous les verbes sont à la forme composée. Cependant il convient de remarquer avec l'auteur que son public n'est pas avec tromper dans le même rapport que la semaine dernière avec sévir. C'est pourquoi je préférerais remplacer la' deuxième phrase par : Les passagers qui ont débarqué hier ont essuyé une tempête.

Ce point une fois acquis, on peut passer à des exemples bilingues :

He has reason to think so.

Il a raison de le penser.

I did not think much of him.

Je n'ai pas beaucoup pensé à lui.

Il est inutile d'insister sur l'écart de sens qui sépare chacune de ces phrases anglaises de la phrase française de même structure. La preuve est faite que la traduction littérale, souvent inélégante et peu idiomatique, entraîne parfois un contresens.

Une fois convaincus de cette vérité, les étudiants peuvent s'exercer à donner de phrases spécialement choisies une traduction idiomatique qui utilise une autre contexture que celle de l'original. Le cas échéant, cet exercice peut s'accompagner d'une ou deux applications de la technique du découpage qui permet de s'assurer que tous les éléments de sens de l'énoncé original se retrouvent dans la traduction et s'y retrouvent seuls. L'existence même de cette technique suppose un ensemble d'analogies et de différences aussi bien au niveau des structures qu'à celui des significations et des valeurs, et cet ensemble constitue la matière de la linguistique différentielle, dont il convient de délimiter les secteurs.

On peut en distinguer quatre : la phonétique, la lexicologie, la grammaire et la stylistique, cette dernière étudiant des faits de prosodie, de lexicologie et de grammaire. Je laisse de côté la phonétique qui est une discipline à part, à l'exception, toutefois, de l'accent de phrase qui constitue souvent en anglais un élément du message et peut être assimilé aux structures grammaticales. 
La différence entre grammaire et stylistique n'est pas arbitraire. Il est exact qu'un fait de langue peut relever des deux, mais stylistique et grammaire sont deux réalités distinctes même quand elles portent sur un même exemple. Dans la traduction de DO NOT ENTER, avis placé à l'entrée d'une rue, les raisons pour lesquelles il ne convient pas de dire «N'entrez pas » relèvent de la stylistique : d'abord on n'entre pas dans une rue, bien que l'on puisse parler de l'entrée d'une rue; on sort dans la rue, venant d'une maison, et si l'on vient d'une autre rue ou d'une place, on s'engage dans une rue. L'énoncé fort improbable $\ll \mathrm{Ne}$ vous engagez pas dans cette rue » respecte la propriété des termes, mais n'est pas adapté à la situation. La langue des avis, en français, est impersonnelle, elle utilise l'infinitif de préférence à l'impératif. On pourrait concevoir : « Ne pas s'engager dans cette rue $»$, mais l'usage administratif emploie rarement le démonstratif. Nous sommes d'ailleurs dans un domaine où il ne s'agit pas tant de traduire que de se demander ce qu'on dit dans l'autre langue en pareil cas. On dit SENS INTERDIT.

On vient de voir que la langue administrative préfère l'infinitif à l'impératif. Cela nous amène à rappeler que l'anglais ne peut pas utiliser l'infinitif actif comme jussif. C'est un fait de structure, et la différence ainsi dégagée entre les deux langues relève de la grammaire et non de la stylistique. On sait que la grammaire étudie surtout les servitudes, tandis que la stylistique étudie les options, ou si l'on préfère, l'utilisation des ressources de la langue en fonction des niveaux de langue et des situations. Cette distinction s'applique au domaine différentiel. Les cas où deux structures sont possibles dans une langue, alors que l'autre n'en admet qu'une seule des deux, sont des cas privilégiés en linguistique différentielle. Le passif en français peut utiliser l'infinitif aussi bien que l'impératif, mais la valeur stylistique de ces deux formes n'est pas la même. Nous avons donc et un fait de grammaire et un fait de stylistique.

En voici un autre exemple, tiré cette fois de l'anglais : He got into conversation with a man he found himself next to in the reception room. Le français ne peut dire que : à côté de qui il se trouva. L'anglais a le choix entre deux séquences : next to whom he found himself et he found himself next to. Comme on pouvait s'y attendre, la coexistence de ces deux constructions synonymes a engendré une différence stylistique.

La grammaire différentielle telle que je la conçois essaie de se placer à mi-chemin entre les deux langues considérées pour étudier leur comportement sur un point donné. Au lieu de partir des formes, elle part des concepts pour décrire leur réalisation grammaticale. Par exemple, elle pourrait partir de would et noter trois emplois importants de cette forme verbale pour marquer le conditionnel, l'itératif et la volonté ; ou encore signaler que should peut, dans certains cas, se rendre par le présent ou le futur de devoir et non par le conditionnel. Mais, pour prendre le dernier cas comme exemple, elle préfère distinguer entre la recommandation, qui peut se rendre en français par le conditionnel de devoir et le règlement qui exige le présent ou le futur. Cela permet de marquer la différence entre $I l$ devrait travailler davantage et Les candidats doivent faire 
parvenir leur demande avant le 30 octobre. L'anglais peut dire should dans les deux cas. C'est également grâce aux analyses de la grammaire différentielle qu'on peut expliquer aux Franco-Canadiens pourquoi on ne peut dire «marcher au bureau » dans l'état actuel du français, et démonter, en quelque sorte la mécanique de la faute qu'a faite le Soleil dans son numéro du mardi 29 septembre en titrant à la page 13 : «Québec augmentera son amende à $\$ 25$ » (pour avoir «brûlé » un signal de circulation). Augmenter, tout comme marcher, est un verbe de mouvement et non de destination. Le chiffre de 25 dollars est, en fait, une destination au même titre que le bureau, et il ne peut être, dans ce contexte, que le complément d'un verbe de destination, qui est porter : Québec portera son amende à $\$ 25$. En anglais, to increase peut être aussi bien verbe de destination que verbe de mouvement, d'où les deux constructions possibles increased by 15 dollars, increased to 25 dollars.

C'est l'un des bienfaits de la linguistique différentielle dans les trois secteurs considérés ici, qu'elle nous oblige à réfléchir au mécanisme des langues en présence et qu'elle nous fait faire sur l'une ou l'autre des découvertes que nous ne ferions sans doute pas si nous bornions notre étude à une seule. Ainsi le verbe prendre est « vectoriel ", tandis que to take est ambivalent et signifie aussi bien porter que prendre. C'est ce que n'ont pas vu les trois traducteurs du Cahier gris de Martin du Gard, qui en traduisant séparément et avec des mots différents ce simple membre de phrase : il reprend entre ses lèvres son bout de cigarette, se sont trompés tous les trois sur la direction du geste et on rendu prendre respectivement par to put back, to set back et to replace, ce qui veut dire qu'ils y ont vu un point d'aboutissement, et non un point de départ comme c'est le cas. Je dois avouer que je n'ai moi-même réfléchi à cette ambivalence du verbe to take que lorsque l'exemple en question m'a été signalé il y a vingt-cinq ans, par l'une de mes étudiantes de l'Université McGill, qui avait consacré sa thèse à l'étude de trois traductions anglaises du Cahier gris ${ }^{1}$.

De semblable façon, préparant récemment une étude sur l'accent de phrase en anglais, je me suis rendu compte, chemin faisant, que l'anglais n'hésite pas à répéter des segments d'énoncé, ce que le français évite de faire; mais cette répétition permet à l'anglais de faire ressortir l'accent de phrase qui tombe sur un mot de la seconde occurrence de l'énoncé : I did not say they were smart, they said they were smart - Ce n'est pas moi qui ai dit qu'ils étaient intelligents, ce sont eux qui le prétendent.

Le rôle de la lexicologie différentielle est de définir les écarts de sens ou de valeur entre les mots de deux langues données qui se correspondent grosso modo, mais dont les aires sémantiques ne coïncident pas. Cette étude trouve sa justification dans les déficiences actuelles des dictionnaires bilingues et elle peut contribuer à en préparer de meilleurs.

Dans le cas de l'anglais et du français, un secteur important de la lexicologie

1. Eleanor J. Laing, Etude critique de trois traductions anglaises du premier volume des "Thibault », de Roger Martin du Gard, thèse de maîtrise de l'Université McGill, 1948. 
différentielle est celui des mots de même origine, de forme analogue et de sens différent que l'on appelle en France depuis 1928 les faux amis. Ce secteur ne semble pas avoir reçu au Canada l'attention qu'il mérite. Peut-être les habitudes invétérées de traduction littérale ont-elles entravé une étude et un enseignement dont l'importance en pays bilingue n'est pourtant pas à démontrer.

Il y a des degrés dans les différences entre faux amis et il y aurait lieu de grouper ceux-ci en catégories. La plus nombreuse serait celle des mots qui n'ont pas la même extension. Ainsi partner et correct ont une aire sémantique plus étendue que celle de partenaire et de correct; brown, à côté de brun, fait figure de générique et rend aussi jaune, marron, bis et bistre. Une autre catégorie est celle dont les sens ne se recouvrent pas du tout : fastidious et fastidieux n'ont rien de commun, eventual est complètement séparé de éventuel. Parfois la différence réside dans la construction du mot : to result a bien le sens de résulter, mais il peut avoir un complément d'effet aussi bien que de cause et correspond ainsi à résulter de (to result from) et à aboutir à (to result into). Familier se dit de la chose qu'on connaît bien, mais non de la personne qui connaît bien cette chose : I am familiar with... se rend donc par cela m'est familier. Certains faux amis relèvent de la stylistique plutôt que de la sémantique; ils sont séparés par des différences de niveaux de langue ou de fréquence. Un traducteur doit savoir que to discuss, to mention, to notify et to prohibit sont plus fréquents en anglais que ne le sont en français les verbes qui leur correspondent par la forme.

Cette liste pourrait s'allonger indéfiniment. Il est plus important d'insister sur l'importance du phénomène. L'une des tâches de la linguistique différentielle est de définir avec plus de précision que ne le font les dictionnaires tout ce qui fait que des mots jugés équivalents ne le sont pas absolument. Dissertation évoque en français le cours de littérature, et en anglais l'exigence principale du doctorat. Dans un domaine donné, il n'y a qu'un gouverneur et un seul. Governor s'emploie de la même façon, mais il s'applique aussi aux membres de certains conseils. Cet emploi est inconnu du français universel et représente l'un des anglicismes canadiens les plus tenaces. La solde est la rémunération du militaire de carrière, mais non du fonctionnaire civil. Il se trouve que pay est à cheval sur les deux domaines, d'où l'emploi franco-canadien de solde au sens de traitement (congé sans solde). Tant que les élèves traducteurs ne sont pas exercés systématiquement à reconnaitre ces différences de sens et d'emploi, ils se font inconsciemment les complices de l'anglicisation du français.

Ce genre d'étude doit se poursuivre à propos de mots qui ne sont pas apparentés ou qui du moins ne présentent pas de similitude de forme susceptible d'induire en erreur. Le jeune francophone qui apprend l'anglais met très tôt en équation road et route, jusqu'au jour où il va en pays anglophone et remarque que certaines rues s'appellent road : Tottenbam Court Road à Londres, Queen Mary Road à Montréal. Sans doute peut-on alléguer que, historiquement, certaines de ces rues ont d'abord été des routes; il n'en reste pas moins que le mot route ne peut s'appliquer à une voie située à l'intérieur d'une ville. Ces différences paraissent négligeables. Rendons-nous compte, cependant, qu'elles peuvent donner lieu à des erreurs de traduction. Un livre de Kipling doit son 
titre français (Simples Contes des collines) à l'ignorance du traducteur qui a cru que tous les hills anglais sont des collines. L'un des avantages de la linguistique différentielle, c'est qu'on peut en faire partout. Ainsi à bord d'un avion d'Air Canada, où les annonces sont bilingues, on peut se rendre compte pour la première fois que prochainement est affaire de jours et non de minutes, ce qui explique le caractère insolite de l'avis : « Nous atterrirons prochainement à Orly ». Il ne semble pas que nos répertoires fassent état de cette restriction de sens qu'il est tout de même utile de connaître si l'on veut traduire correctement.

Faire cette remarque, c'est amorcer le procès de nos dictionnaires bilingues. Dans un article de Langages ( $\mathrm{n}^{\circ} 19$, septembre 1970), j'ai essayé d'analyser les déficiences des principaux dictionnaires français-anglais et anglais-français, en tenant compte, bien entendu, du peu de place dont ils disposent et en posant le principe qu'ils doivent éclairer l'usager aussi complètement que possible pour chaque mot qu'ils décident de retenir. L'étendue de la nomenclature dépend finalement $\mathrm{d} u$ format, mais devrait être subordonnée au traitement des articles.

Les déficiences qu'on peut relever tiennent surtout à l'absence de catégories sémantiques telles que l'extension, la dichotomie des sens propre et figuré et celle des valeurs intellectuelle et affective, qui, dans le cas des adjectifs, recoupent la dichotomie de l'inhérence et de la relation. Il est facile de mettre des réalités concrètes sous ces termes techniques. L'anglophone qui doit traduire room a besoin qu'on lui explique que ce mot est tantôt générique (auquel cas il se traduit par pièce), tantôt spécifique, et alors il se rend, suivant le contexte, par chambre, salle, bureau. Le même problème se pose pour le francophone qui a à traduire pétrole, car ce terme peut lui aussi être générique ou spécifique; dans le premier cas, il correspond à petroleum ou à oil, et dans le second à kerosene. Aucun des dictionnaires examinés ne montre clairement que le mot cutlery a plus d'extension que coutellerie, puisqu'il s'applique aussi aux couteaux, fourchettes et cuillers que l'on dispose sur la table au moment des repas, et que nos archives peuvent être commerciales (en anglais records) aussi bien qu'historiques (en anglais archives). Voilà pour l'extension.

En ce qui concerne les autres catégories sémantiques, rappelons que héritage a le sens propre et que son paronyme anglais heritage s'emploie surtout au figuré, que charitable n'est qu'un adjectif d'inhérence en français, tandis qu'en anglais il peut aussi être adjectif de relation et se traduit alors par de bienfaisance. Inversement, unique est seulement affectif en anglais, alors qu'en français il peut être intellectuel aussi bien qu'affectif, et c'est pourquoi fils unique se rend en anglais par only son. J'ai déjà fait allusion dans META (numéro de septembre 1969) à ces classements de sens, qui relèvent en partie de la stylistique.

La lexicologie différentielle devrait aussi se préoccuper de dégager certains caractères des deux vocabulaires qu'elle rapproche. C'est particulièrement utile pour le traducteur, qui est parfois tenté de demander à la langue d'arrivée plus qu'elle ne peut donner. Ainsi c'est une caractéristique du français d'avoir un vocabulaire beaucoup moins motivé que celui de l'anglais. Nous disons une cravate saumon et tout le monde comprend qu'il s'agit de sa couleur; point n'est besoin d'être aussi explicite que salmon-coloured. Cette absence relative de moti- 
vation explique l'importance du contexte en français, car c'est le contexte qui nous permet de traduire chambre à coucher par bedroom ou par bedroom set ( «set de chambre» est une surtraduction), épicerie par grocery store ou grocery trade, et de choisir, pour traduire chimney, l'un des quatre ou cinq équivalents de ce terme.

Dans le même ordre d'idées, il peut être utile d'opposer les mots très généraux à ceux qui au contraire sont très particuliers. Il n'y a pas de termes aussi spécifiques que margelle, perron ou cran en anglais, et que to remit, to assay, wand en français. Nous dirons que ces mots sont surcaractérisés, tandis que bord et dent en français, car et handle en anglais sont sous-caractérisés, et de ce fait ont plusieurs équivalents dans l'autre langue. Ainsi handle correspond, suivant le cas, à manche, anse, manette, manivelle.

Les exemples que nous avons passés en revue avaient pour but de donner, pour chacun des trois secteurs, une idée des possibilités d'étude et de leurs applications pratiques. Le moment est venu d'examiner la place de cette discipline parmi les préoccupations professionnelles des traducteurs.

Aujourd'hui, il semble que le grand problème de la traduction soit d'ordre terminologique. Les textes techniques, qui constituent une part importante de la matière à traduire, posent des difficultés qui tendent à accaparer l'attention des traducteurs. Cette préoccupation est parfaitement justifiée, mais elle ne doit pas faire oublier le reste. Quand on lit des traductions en qualité de réviseur, on constate que la syntaxe laisse parfois à désirer; on relève aussi des erreurs qui tiennent à des ignorances de vocabulaire, par exemple dans la catégorie des faux amis. Récemment, dans un certain rapport traduit en anglais, le mot éventuel, employé à deux ou trois reprises, a été chaque fois traduit par eventual et on a l'impression que le traducteur ne s'y est pas arrêté un instant. L'idée ne lui est sans doute pas venue que ces deux termes puissent ne pas coïncider.

Il est exact que la linguistique différentielle, telle qu'elle a été présentée ci-dessus, n'est pas destinée à résoudre les problèmes terminologiques, dont la solution exige la collaboration des spécialistes et des linguistes. Mais on ne traduit pas que des termes techniques, on traduit aussi les mots qui les entourent, ainsi que le message qui résulte de l'agencement de ces mots et fait état de certaines valeurs affectives du langage. Si les cadres que propose la lexicologie différentielle sont surtout destinés au vocabulaire général, ce vocabulaire est une des assises de cet édifice de vocabulaire technique que chaque traducteur spécialisé doit construire pour son usage personnel. De sorte qu'aussi bien pour structurer un message sans trahir la pensée de l'auteur que pour rendre compte exactement des réalités techniques souvent pensées dans la langue de départ, il faut au préalable une bonne préparation linguistique portant d'abord sur chaque langue prise séparément et ensuite sur une comparaison méthodique de leurs réalisations respectives.

On peut même aller jusqu'à dire que la linguistique différentielle fournit aussi une thérapeutique pour ces maux dont sont souvent affligés les traducteurs 
en pays bilingues, et qui sont dus essentiellement à la peur des mots ; une scrupulosité maladive tend en effet à créer de faux problèmes et à négliger ceux qui se posent réellement. Cette insécurité linguistique, là où elle existe, tient à ce qu'on ne sait pas au juste où une langue finit et où l'autre commence. C'est la tâche essentielle de la linguistique différentielle de marquer la ligne de faîte et d'éclairer les versants.

JEAN DARBELNET 